\title{
Geometric Control of Particle Manipulation in a Two-Dimensional Fluid
}

\author{
Yizhar Or, Joris Vankerschaver, Scott D. Kelly, Richard M. Murray and Jerrold E. Marsden
}

\begin{abstract}
Manipulation of particles suspended in fluids is crucial for many applications, such as precision machining, chemical processes, bio-engineering, and self-feeding of microorganisms. In this paper, we study the problem of particle manipulation by cyclic fluid boundary excitations from a geometric-control viewpoint. We focus on the simplified problem of manipulating a single particle by generating controlled cyclic motion of a circular rigid body in a two-dimensional perfect fluid. We show that the drift in the particle location after one cyclic motion of the body can be interpreted as the geometric phase of a connection induced by the system's hydrodynamics. We then formulate the problem as a control system, and derive a geometric criterion for its nonlinear controllability. Moreover, by exploiting the geometric structure of the system, we explicitly construct a feedback-based gait that results in attraction of the particle towards the rigid body. We argue that our gait is robust and model-independent, and demonstrate it in both perfect fluid and Stokes fluid.
\end{abstract}

\section{INTRODUCTION}

A variety of practical tasks - ranging from the sorting and filtering of airborne contaminants for public safety [14] to the mechanical testing of macromolecules like DNA [29] to the assisted fertilization of human ova with immotile sperm [10] to the stirring of chemical processes [1] — involve the manipulation of particles suspended in fluids. Depending on the application, direct mechanical manipulation of the particles in question can be impractical because of their number (as with environmental pollutants) or their delicacy (as with living cells). Current alternatives addressing one or the other of these issues have problems of their own: laserbased systems like optical tweezers [4], for instance, are relatively gentle but require translucent media (and closedloop control) for practical implementation.

Examples of particle manipulation through periodic fluid boundary excitation are present throughout the natural world, suggesting a class of simply realized engineered systems with diverse applications. The ingestion of nutrients by many aquatic organisms hinges, for instance, on the entrainment of these nutrients in flow structures maintained by vibrating appendages. Among the simplest such structures is the

Y. Or, J. Vankerschaver, R. Murray and J. Marsden are with the Control and Dynamical Systems Department, California Institute of Technology, Pasadena, CA 91125, USA.

J. Vankerschaver is also with the Department of Mathematical Physics and Astronomy, Ghent University, B-9000 Ghent, Belgium. S. Kelly is with the Department of Mechanical Engineering and Engineering Science, University of North Carolina at Charlotte, Charlotte, NC 28223-0001, USA.

YO is supported by a Fulbright Postdoctoral Fellowship and a Bikura Postdoctoral Scholarship of the Israeli Science Foundation. JV is supported by a Postdoctoral Fellowship of the Research Foundation — Flanders (FWO-Vlaanderen). toroidal feeding vortex of the ciliated protozoan Vorticella [30], depicted in the video at [26]. Compare this video to that at [31], which shows the toroidal vortex surrounding a vibrating carbon-fiber probe penetrating a droplet of water in which a large number of forty-micron polymer beads are suspended [32]. The sustained excitation of abrasive particles in this manner has proven an effective strategy for the precision machining of brittle surfaces [24]. In a different implementation, a microfluidic device has been developed for manipulating cells by controlling external electric fields and using vision-based feedback control [3]

The mixing properties of the ciliary motion described above have recently been studied experimentally [23], and efforts to develop artificial cilia are ongoing in the MEMS community [12], but a consistent framework has not been established for studying the dynamics and control of such systems - let alone the broader class of systems in which cyclic boundary motions of arbitrary geometry are invoked to manipulate particles suspended in fluids on arbitrary physical scales. We initiate the development of such a framework in the present paper.

We focus the present discussion on a planar system resembling the oscillating probe from [31] in cross section, as shown in Fig. 1. In modeling the coupling between the actuated motion of the shaded cylinder and the motion of the surrounding fluid, we assume the fluid to be inviscid. This assumption represents a natural first step toward more complicated flow models, but does not necessarily restrict the relevance of our results to the context of conservative systems, and we demonstrate the effectiveness of a control strategy derived with the help of this model in the opposite extreme of Stokes flow. Indeed, prior work by the authors in the context of locomotion - conceptually, the mirror image of fluid transport through boundary motion - has explored both the formal parallelism between ideal flow and Stokes flow [16] and the correlation between optimally propulsive boundary motions in ideal flows and in flows dominated by dynamic vortex shedding [17].

Contributions: The aims and achievements of this paper are twofold. First and foremost, we investigate the problem of a particle moving in the fluid field generated by an actuated rigid body from a control-theoretic point of view. We show that the system is nonlinearly controllable and design a gait for the rigid body that succeeds in attracting the particle. Despite the low dimensionality of the problem, this is by no means an easy task: naive gaits move the particle on a circle but do not manage to bring it closer. By exploiting the geometric structure of the control system, we design 


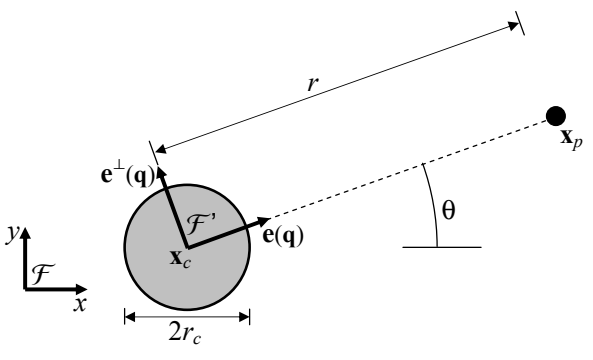

Fig. 1. The cylinder-particle system

a feedback-based gait that succeeds in doing so. Our gait has the added advantage of being model-independent in the sense that, while the main body of the text is concerned with rigid bodies in ideal, inviscid flows, the same strategy also works for other types of control systems possessing similar geometric structure, as demonstrated for the case of two rigid bodies moving in Stokes flow.

Secondly, we argue that this example fits into a broader class of control systems, whose characteristic property is that their configuration space has the structure of a fiber bundle. Using some elementary tools from differential geometry and nonlinear control theory, we show that the particle manipulation problem can be understood in terms of a connection on the fiber bundle, so that the drift in the particle location after one cyclic change in the position of the rigid body can be described by the geometric phase (or holonomy) of this connection. While geometric phases have been used in control theory to great effect before (see [22], [8], [27], [18], [15]), this approach has traditionally been confined to the case of locomotion systems, that enjoy some additional symmetry properties due to the fact that the output variables are group-valued. The class of control systems that we consider here is distinct from that, but the geometric structure still allows for significant simplifications: we prove a new controllability criterion which is equivalent to the classical Chow theorem, but uses objects on the space of output variables only, leading to a significant reduction in dimensionality.

\section{PROBLEM DESCRIPTION}

Consider a rigid, circular body of radius $r_{c}$ moving in an inviscid, incompressible two-dimensional fluid which is at rest at infinity. Physically, this ideal model corresponds to the case of flow with large Reynolds number, where the hydrodynamics is governed primarily by inertial effects, whereas the effect of viscous drag is negligible [20]. As in Fig. 1, we denote the position of the center of the body by $\mathbf{x}_{c}$. Our aim is to design bounded, cyclic gaits for the motion of the rigid body that bring a distinguished particle, whose location is denoted by $\mathbf{x}_{p}$, closer to the body.

\section{A. Potential Flow}

Consider an inviscid, incompressible fluid with Eulerian velocity field $\mathbf{v}(\mathbf{x}, t)$. When the curl $\nabla \times \mathbf{v}$ vanishes, the fluid is said to be irrotational. For two-dimensional flows, this implies that the Euler equations describing $\mathbf{v}$ are reduced to solving a Laplace equation, as we recall below. For more information about irrotational fluid dynamics, see [20].

In the case of an irrotational fluid, the velocity field can be written as a gradient of a potential function $\mathbf{v}=\nabla \phi$, where $\phi$ is the solution of Laplace's equation $\nabla^{2} \phi=0$ with Neumann boundary conditions given by the fact that the normal velocity of the fluid should be equal to the normal velocity of the points on the boundary of the body:

$$
\mathbf{n} \cdot \nabla \phi=\left(\boldsymbol{\omega} \times\left(\mathbf{x}-\mathbf{x}_{c}\right)+\dot{\mathbf{x}}_{c}\right) \cdot \mathbf{n} \quad \text { on } \partial \mathcal{B} \text {, }
$$

together with the fact that $\phi$ goes to zero sufficiently fast as $\|\mathbf{x}\| \rightarrow \infty$. Here $\boldsymbol{\omega}$ and $\dot{\mathbf{x}}_{c}$ are the angular and linear velocity of the rigid body, and $\mathbf{n}$ is the unit normal to the boundary of the body.

Because of the linear dependence of $\phi$ on $\boldsymbol{\omega}=\omega \mathbf{e}_{z}$ and $\dot{\mathbf{x}}_{c}=\dot{x}_{c} \mathbf{e}_{x}+\dot{y}_{c} \mathbf{e}_{y}, \phi$ can be expressed as $\phi=\phi_{\omega} \omega+$ $\phi_{x} \dot{x}_{c}+\phi_{y} \dot{y}_{c}$, where $\phi_{\omega}, \phi_{x}$ and $\phi_{y}$ are the elementary velocity potentials. For a circular rigid body of radius $r_{c}$, these potentials are given by $\phi_{\omega}=0$ and

$$
\phi_{x}(\mathbf{x})=-r_{c}^{2} \frac{x-x_{c}}{\left\|\mathbf{x}-\mathbf{x}_{c}\right\|^{2}}, \quad \phi_{y}(\mathbf{x})=-r_{c}^{2} \frac{y-y_{c}}{\left\|\mathbf{x}-\mathbf{x}_{c}\right\|^{2}},
$$

where $\mathbf{x}=(x, y)$ and $\mathbf{x}_{c}$ is the location of the center of the cylinder. For future reference, we also introduce the associated elementary stream functions $\psi_{x}$ and $\psi_{y}$ as the harmonic conjugates of $\phi_{x}$ and $\phi_{y}$ :

$$
\frac{\partial \phi_{x}}{\partial x}=\frac{\partial \psi_{x}}{\partial y} \quad \text { and } \quad \frac{\partial \phi_{x}}{\partial y}=-\frac{\partial \psi_{x}}{\partial x},
$$

and similarly for $\psi_{y}$.

\section{B. Velocity of Fluid Particles}

We now consider the motion of a single massless particle $\mathbf{x}_{p}(t)$ in the irrotational flow field of a moving rigid body with center of mass $\mathbf{x}_{c}(t)$. As the particle is advected by the fluid flow (i.e. it does not influence the fluid, but is simply swept along), its velocity is equal to the fluid velocity at that point: $\dot{\mathbf{x}}_{p}=\mathbf{v}\left(\mathbf{x}_{p}(t), t\right)$. The time-dependence of the fluid velocity field is due to the motion of the rigid body.

The equations of motion for the particle can be made more explicit by using the expressions in (1) for the velocity potentials:

$$
\dot{\mathbf{x}}_{p}=\mathbf{A}\left(\mathbf{x}_{p}, \mathbf{x}_{c}\right) \cdot \dot{\mathbf{x}}_{c}
$$

Here, the matrix $\mathbf{A}\left(\mathbf{x}_{p}, \mathbf{x}_{c}\right)$ is given by

$$
\mathbf{A}\left(\mathbf{x}_{p}, \mathbf{x}_{c}\right)=\frac{r_{c}^{2}}{\left(X^{2}+Y^{2}\right)^{2}}\left(\begin{array}{cc}
-X^{2}+Y^{2} & 2 X Y \\
2 X Y & X^{2}-Y^{2}
\end{array}\right)
$$

where $(X, Y)$ denote the components of the relative position vector $\mathbf{x}_{p}-\mathbf{x}_{c}$. Note that the matrix $\mathbf{A}$ depends on the relative position $\mathbf{x}_{p}-\mathbf{x}_{c}$ only. 

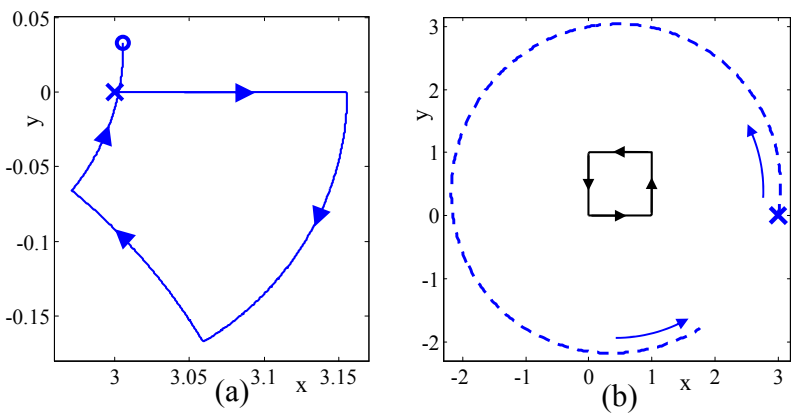

Fig. 2. Particle motion under a square loop motion of the cylinder: (a) Particle trajectory under a single cycle. (b) Particle trajectory (dashed line) under 400 square loops cycles of the cylinder (solid curve).

\section{Formulation as a Control Problem}

The problem we study in this paper is controlling the motion of the cylinder $\mathbf{x}_{c}$ in a way that induces a desired motion of the particle $\mathbf{x}_{p}$. More precisely, we wish to move the cylinder in arbitrarily small bounded closed loops, and generate attraction of the particle towards it. The input of the system is the velocity of the cylinder,

$$
\dot{\mathbf{x}}_{c}=\mathbf{u},
$$

and the output is the position of the particle $\mathbf{x}_{p}$. Equations (3) and (5) describe the problem as a driftless nonlinear control system [6], [28]. In order to check the local controllability of the system, one can apply the classical Lie-algebraic test originally developed by Chow [7]. Direct application of this test for our system shows that it is nonlinearly controllable as long as $X^{2}+Y^{2} \neq r_{c}^{2}$, which is satisfied whenever the particle is not strictly in contact with the cylinder's boundary. We will return to this in section VI, where we exploit the geometric structure of the system to develop an alternative controllability criterion.

Simulation Example. Consider the case of a cylinder with radius $r_{c}=1$ whose initial center position is $\mathbf{x}_{c}=(0,0)$, and a particle which is initially located at $\mathbf{x}_{p}=(3,0)$. Fig. 2(a) shows the motion of the particle as a result of the motion of the cylinder on an anti-clockwise square loop with sides of length 1 . The initial point is marked by ' $x$ ', while the end point is marked by ' $\circ$ '. Next, we simulate the motion of the particle under repetition of the square loop of the cylinder's motion for 400 times; see Fig. 2(b). The result is that the particle traces out a circle centered on the origin, without getting any closer to the cylinder. Our particular goal, to attract the particle towards the cylinder, can therefore not be achieved by naively applying simple loops for the cylinder's motion. In the sequel, we analyze the underlying geometric structure of our control system, and present an algorithm for generating a feedback-based gait of periodic motion of the cylinder that will result in a slow drift of the particle towards it.

\section{CONNECTION-THEORETIC DESCRIPTION}

In this section, we make a first step towards constructing gaits, by interpreting the matrix $\mathbf{A}(\mathbf{q})$ in (3) as a connection in a fiber bundle, and showing how the drift in the particle location due to a cyclic change in the location of the rigid body can be viewed as the geometric phase (or holonomy) of this connection. This is distinct from the usual appearance of connections and geometric phases for locomotion systems, which are mechanical control systems defined on a principal fiber bundle [6], [18]. These systems are (locally) of the form $g^{-1} \dot{g}=\mathcal{A}(s) \dot{s}$, where the variables $g$ take values in a Lie group and the matrix $\mathcal{A}(s)$ depends on the input parameters $s$ only. In contrast, the connection matrix $\mathbf{A}\left(\mathbf{x}_{p}, \mathbf{x}_{c}\right)$ in this paper does not have these group-theoretical properties and depends both on the input and the output variables, precluding a standard geometrical approach to motion planning as in [22], [27].

\section{A. Fiber Bundles and Connections}

We now describe how the particle manipulation system (3) and (5) fits into a broader class of control systems. We denote the space of input variables for this class of systems by $B$ and the space of output variables by $F$. To emphasize the link with the particle manipulation problem, we will denote points in $B$ and $F$ by $\mathbf{x}_{c}$ and $\mathbf{x}_{p}$, respectively. However, we emphasize that this setup is applicable to more general problems as well.

The spaces $B$ and $F$ naturally fit together into a differential-geometric structure known as a fiber bundle. For our purposes, it will suffice to define a (trivial) fiber bundle to be a triple $\left(B \times F, B, \pi_{1}\right)$, where $B$ and $F$ are manifolds and $\pi_{1}: B \times F \rightarrow B$ is the projection onto the first factor. The manifold $B$ is called the base space of the bundle and encodes the internal, controlled variables, while $B \times F$ is referred to as the total space, where the fiber $F$ encodes the output variables.

In order to specify the relation between the control parameters and the output variables, we need the concept of a connection. While there are many equivalent ways of defining connections in this context, we use the concept of a horizontal lift [6].

Definition 1: A horizontal lift is a family of linear maps

$$
\mathbf{A}\left(\mathbf{x}_{p}, \mathbf{x}_{c}\right): T_{\mathbf{x}_{c}} B \rightarrow T_{\mathbf{x}_{c}} B \times T_{\mathbf{x}_{p}} F,
$$

for all $\left(\mathbf{x}_{c}, \mathbf{x}_{p}\right) \in B \times F$, such that $T \pi_{1} \circ \mathbf{A}\left(\mathbf{x}_{p}, \mathbf{x}_{c}\right)=\mathrm{id}$, i.e. $\mathbf{A}\left(\mathbf{x}_{p}, \mathbf{x}_{c}\right)$ is the identity on the first factor.

When evaluated on a vector $\dot{\mathbf{x}}_{c}$, the horizontal lift map $\mathbf{A}\left(\mathbf{x}_{p}, \mathbf{x}_{c}\right) \cdot \dot{\mathbf{x}}_{c}$ determines the influence on $\mathbf{x}_{p}$ of an infinitesimal change in $\mathbf{x}_{c}$, given by $\dot{\mathbf{x}}_{c}$. In order to describe cyclic changes in the control variables, we recall the concept of geometric phase of a connection. Let $\gamma: t \mapsto \mathbf{x}_{c}(t)$ be a closed loop in $B$ with $\mathbf{x}_{c}(0)=\mathbf{x}_{c}(1)=\mathbf{x}_{c}$, and choose a point $\mathbf{x}_{p} \in F$. We can then find a unique curve $t \mapsto \mathbf{x}_{p}(t)$ in $F$ such that

$$
\left(\dot{\mathbf{x}}_{c}(t), \dot{\mathbf{x}}_{p}(t)\right)=\mathbf{A}\left(\mathbf{x}_{p}, \mathbf{x}_{c}\right) \cdot \dot{\mathbf{x}}_{c}(t),
$$

satisfying the initial condition $\mathbf{x}_{p}(0)=\mathbf{x}_{p}$. The pair $\left(\mathbf{x}_{c}(t), \mathbf{x}_{p}(t)\right)$ is called the horizontal lift of $\gamma$, based at $\left(\mathbf{x}_{c}, \mathbf{x}_{p}\right) \in B \times F$. Fig. 3 illustrates the geometric phase map associated to a given loop in the base space. 


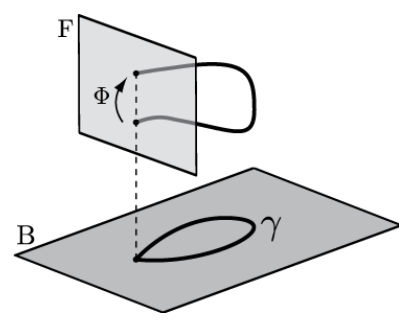

Fig. 3. Illustration of the geometric phase associated to a loop in the base space.

Definition 2: Let $\gamma: t \mapsto \mathbf{x}_{c}(t)$ be a closed loop in $B$. For $\mathbf{x}_{p} \in F$, consider the horizontal lift $\left(\mathbf{x}_{p}(t), \mathbf{x}_{c}(t)\right)$ of that loop, based at $\left(\mathbf{x}_{p}, \mathbf{x}_{c}\right)$. The geometric phase associated to the loop $\gamma$ is the map $\Phi: F \rightarrow F$ associating to each $\mathbf{x}_{p}$ the end point $\mathbf{x}_{p}(1)$ of the curve $\mathbf{x}_{p}(t)$.

Note that the geometric phase map depends only on the geometric shape of the loop $\gamma$, and not on the choice of a time-parametrization of the motion of $\mathbf{x}_{c}(t)$ along $\gamma$.

\section{B. Application to Particle Manipulation}

For the particle manipulation problem, the base space $B$ is the configuration space of the rigid body: $B=\mathbb{R}^{2}$, while the fiber $F=\mathbb{R}^{2}$ is the particle's configuration space. ${ }^{1}$ The matrix $\mathbf{A}$ in (4) defines a connection in this bundle. We now describe some properties of this connection.

a) Geometric Phase: For this connection, the lifted curve $\left(\mathbf{x}_{c}(t), \mathbf{x}_{p}(t)\right)$ associated to a closed loop $\mathbf{x}_{c}(t)$ in the body space has the following interpretation: $\mathbf{x}_{p}(t)$ describes the trajectory of the particle, relative to the body, as the body traces out the curve $\mathbf{x}_{c}(t)$.

b) Volume-preservation: Since the fluid is taken to be incompressible, the geometric phase map $\Phi: \mathbb{R}^{2} \rightarrow \mathbb{R}^{2}$ is volume-preserving, regardless of the trajectory in the base space. This can also be checked directly by noting that the divergence of the right-hand side of (3) vanishes.

\section{GeOMETRIC STRUCTURE OF THE CONTROL SYSTEM}

We now study the geometric structure and symmetries of the control system (3) and (5), by making the following three observations. The first observation is that the connection $\mathbf{A}\left(\mathbf{x}_{p}, \mathbf{x}_{c}\right)$ in (4) depends only on the relative position $\mathbf{q}$, defined as $\mathbf{q}=\mathbf{x}_{p}-\mathbf{x}_{c}$. The control system can then be reformulated in terms of $\mathbf{q}$ as

$$
\left\{\begin{array}{l}
\dot{\mathbf{q}}=\mathbf{B}(\mathbf{q}) \mathbf{u} \\
\dot{\mathbf{x}}_{c}=\mathbf{u}
\end{array}\right.
$$

where $\mathbf{B}(\mathbf{q})=\mathbf{A}(\mathbf{q})-\mathbf{I}$, and $\mathbf{I}$ is the $2 \times 2$ identity matrix. The second observation, which exploits the circular symmetry of the cylinder, is that equation (7) is invariant under simultaneous rotation of $\mathbf{q}, \dot{\mathbf{q}}$ and $\mathbf{u}$, as follows. Let $\mathbf{R} \in S O(2)$

\footnotetext{
${ }^{1}$ More precisely, one needs to restrict $F$ to the region exterior to the rigid body, but this caveat does not affect our treatment.
}

be a rotation matrix in the plane. Then $\dot{\mathbf{q}}=\mathbf{B}(\mathbf{q}) \mathbf{u}$ implies $\mathbf{R} \dot{\mathbf{q}}=\mathbf{B}(\mathbf{R q}) \mathbf{R u}$. Therefore, $\mathbf{B}(\mathbf{q})$ satisfies

$$
\mathbf{B}(\mathbf{q})=\mathbf{R B}\left(\mathbf{R}^{T} \mathbf{q}\right) \mathbf{R}^{T}
$$

The third observation is that the control system (7) is invariant under reflection with respect to the particle-cylinder line, whose direction is $\mathbf{q}$, so that

$$
\mathbf{B}(\mathbf{q})=\mathbf{P}(\mathbf{q}) \mathbf{B}(\mathbf{q}) \mathbf{P}(\mathbf{q}),
$$

where $\mathbf{P}=\mathbf{I}-2 \mathbf{e}^{\perp}(\mathbf{q}) \mathbf{e}^{\perp}(\mathbf{q})^{T}$ is the reflection operator about the direction of $\mathbf{q}$. Here we have introduced

$$
\mathbf{e}^{\perp}(\mathbf{q})=\mathbf{J e}(\mathbf{q}), \quad \mathbf{e}=\frac{\mathbf{q}}{\|\mathbf{q}\|}, \quad \text { and } \quad \mathbf{J}=\left(\begin{array}{rr}
0 & -1 \\
1 & 0
\end{array}\right) .
$$

We now define a rotating reference frame $\mathcal{F}^{\prime}$ which is aligned with the direction of $\mathbf{q}$ (see Fig. 1). Let $\mathbf{R}(\theta)$ be the rotation matrix from the frame $\mathcal{F}^{\prime}$ to the worldfixed frame $\mathcal{F}$, where $\theta=\tan ^{-1}(Y / X)$ and $(X, Y)$ are the components of $\mathbf{q}$ expressed in the frame $\mathcal{F}$. Define $\mathbf{q}^{\prime}=\mathbf{R}(\theta)^{T} \mathbf{q}, \dot{\mathbf{q}}^{\prime}=\mathbf{R}(\theta)^{T} \dot{\mathbf{q}}$, and $\mathbf{u}^{\prime}=\mathbf{R}(\theta)^{T} \mathbf{u}$, which are the vectors $\mathbf{q}, \dot{\mathbf{q}}$ and $\mathbf{u}$ expressed in the frame $\mathcal{F}^{\prime}$. By construction, we have that $\mathbf{q}^{\prime}=\left(\begin{array}{ll}0 & r\end{array}\right)^{T}$, where $r=$ $\|\mathbf{q}\|$. Defining $\mathbf{B}^{\prime}(r)=\mathbf{B}\left(\mathbf{q}^{\prime}\right)$, for given $\mathbf{q}, \mathbf{B}(\mathbf{q})$ can be obtained from $\mathbf{B}^{\prime}(r)$ according to (8). Note that $\mathbf{B}^{\prime}(r)$ depends only on the distance $r$ between the cylinder and the particle. Moreover, augmenting this observation with the relation (9), we conclude that $\mathbf{B}^{\prime}(r)$ is diagonal. Note that our geometric interpretation is general, and applies to any system of the form (7) describing the interaction between two planar bodies with circular symmetries. Nevertheless, in our specific example given in (3) and (4), direct calculation gives $\mathbf{B}^{\prime}(r)=\operatorname{diag}\left\{B_{11}^{\prime}(r), B_{22}^{\prime}(r)\right\}$, where

$$
B_{11}^{\prime}(r)=\frac{r_{c}^{2}}{r^{2}}-1 \text { and } B_{22}^{\prime}(r)=-\frac{r_{c}^{2}}{r^{2}}-1 .
$$

The radial and tangential motions. We now characterize two principal motions for this system, namely, radial and tangential motions, implied by the diagonality of $\mathbf{B}^{\prime}(r)$. These two principal motions play a key role in the algorithm we present for gait generation. Radial motion is a motion in which the input velocity $\mathbf{u}=\dot{\mathbf{x}}_{c}$ is directed along $\mathbf{q}$. In this case, the motion of $\mathbf{x}_{c}$ and $\mathbf{x}_{p}$ is one-dimensional, and depends only on the separation distance $r$. Taking $\mathbf{u}=\mathbf{e}(\mathbf{q})$, $r$ satisfies the differential equation

$$
\dot{r}(t)=B_{11}^{\prime}(r(t)) \text {. }
$$

This equation is separable, and for our specific case given in (10), the solution $r(t)$ with initial condition $r(0)=r_{0}$ is obtained from the implicit equation

$$
\frac{r_{c}}{2}\left(\log \frac{r+r_{c}}{r-r_{c}}-\log \frac{r_{0}+r_{c}}{r_{0}-r_{c}}\right)+r_{0}-r=t .
$$

For large distances $r \gg r_{c}$, the solution has a constant slope of -1 , indicating that the cylinder is moving while the particle stands still. For small initial distance $r_{0}$, the solution $r(t)$ approaches $r_{c}$ asymptotically. Therefore, catching the particle by directly "chasing" it is possible, but requires large 
motions, whereas here we focus on manipulating the motion of $\mathbf{x}_{p}$ by generating bounded motions of $\mathbf{x}_{c}$.

The tangential motion is generated by applying input velocity $\mathbf{u}=\mathbf{e}^{\perp}(q)$, which is constantly perpendicular to q. Under this motion, the distance $\left\|\mathbf{x}_{p}-\mathbf{x}_{c}\right\|$ remains fixed at $r=r_{0}$. Moreover, it can be shown that under this input, $\mathbf{x}_{c}$ and $\mathbf{x}_{p}$ are moving along two concentric circular arcs, where the radius of the circular arc formed by the motion of $\mathbf{x}_{c}$ is $R=\frac{r_{0}}{\left|B_{22}^{\prime}\left(r_{0}\right)\right|}$.

\section{THE FEEDBACK-BASEd GAIT}

We now present a novel algorithm for generating a gait for this system, which results in a decrease of the distance between $\mathbf{x}_{c}$ and $\mathbf{x}_{p}$. The gait makes use of the two principal motions described above in a way that generates a closed loop. We prove that the gait results in attraction of the particle $\mathbf{x}_{p}$ to the body $\mathbf{x}_{c}$, and demonstrate the resulting motion via numerical simulations. Though the gait requires feedback, it does not depend on details of the specific model of the system, hence it is robust.

\section{A. The TRTR gait procedure}

The $T R T R$ gait consists of four steps, alternating between tangential and radial motions. The input velocity $\mathbf{u}=\dot{\mathbf{x}}_{c}$ in each step is described as follows.

A. Apply input $\mathbf{u}=\mathbf{e}^{\perp}(\mathbf{q})$ for a time $t_{1}$.

B. Apply input $\mathbf{u}=\mathbf{e}(\mathbf{q})$ for a time $t_{2}$.

C. Apply input $\mathbf{u}=-\mathbf{e}^{\perp}(\mathbf{q})$, until reaching a time at which the initial position $\mathbf{x}_{c}(0)$ and the current positions $\mathbf{x}_{c}$ and $\mathbf{x}_{p}$ all lie on a common line.

D. Apply input $\mathbf{u}=-\mathbf{e}(\mathbf{q})$ until $\mathbf{x}_{c}$ reaches its initial position $\mathbf{x}_{c}(0)$.

The $T R T R$ gait is explained as follows. In step $\mathrm{A}, \mathbf{x}_{c}$ and $\mathbf{x}_{p}$ are moving along two concentric arcs about a centerpoint $O_{1}$, while the distance between them is kept fixed at $r=r_{0}$. In step $\mathrm{B}, \mathbf{x}_{c}$ and $\mathbf{x}_{p}$ are moving along the line connecting them, and the distance between them is decreased to $r_{2}$. Then in step $\mathrm{C}, \mathbf{x}_{c}$ and $\mathbf{x}_{p}$ are again moving along concentric circular arcs with direction reversed from step A. A key fact is that the center point of these arcs $O_{3}$ is now shifted from $O_{1}$ by a distance $\Delta_{o}$, and the arcs' radii for $\mathbf{x}_{c}(t)$, denoted $R_{1}$ and $R_{3}$, are unequal. When step $\mathrm{C}$ comes to an end, the points $\mathbf{x}_{p}$, $\mathbf{x}_{c}$ and $\mathbf{x}_{c}(0)$ are lying along a common line. In step $\mathrm{D}, \mathbf{x}_{c}$ moves along this line for a time $t_{4}$, until it reaches its original position $\mathbf{x}_{c}(0)$. The distance $r$ is increasing in this step. Once this gait is completed, $\mathbf{x}_{c}$ returns to its starting point, whereas $\mathbf{x}_{p}$ does not. This results in a shift of both the distance $r$ and the angle $\theta$, which is precisely the geometric phase associated with the gait. The geometric phase depends on the times $t_{1}$ and $t_{2}$, which are design parameters of the gait, but has no explicit expression, as it involves the implicit solution of Eq. (11) describing the radial motion. Denoting by $\Phi\left(r_{0}, t\right)$ the solution of (11) at time $t$ under initial condition $r(0)=r_{0}$, the following lemma gives the implicit expression for $r_{e n d}$, which is the final distance $r$ under the TRTR gait. The proof of this lemma, as well as the expression for the shift in $\theta$, are not given here due to space constraints.
Lemma 1: Consider a control system of the form (7), that satisfies the invariance properties (8) and (9). For given initial distance $r(0)=r_{0}$, the final distance $r_{\text {end }}$ under the TRTR gait with times $t_{1}$ and $t_{2}$ is given by

$$
\begin{aligned}
& r_{\text {end }}=\Phi\left(r_{0}, t_{2}-t_{4}\right), \quad \text { where } \\
& t_{4}=\sqrt{\left(\Delta_{o}+R_{1} \cos \theta_{1}\right)^{2}+\left(R_{1} \sin \theta_{1}\right)^{2}}-R_{3} \\
& \Delta_{o}=t_{2}+R_{3}-R_{1}, \theta_{1}=\frac{t_{1}}{R_{1}} \\
& R_{1}=\frac{r_{0}}{\left|B_{22}^{\prime}\left(r_{0}\right)\right|}, R_{3}=\frac{r_{2}}{\left|B_{22}^{\prime}\left(r_{2}\right)\right|}, r_{2}=\Phi\left(r_{0}, t_{2}\right) .
\end{aligned}
$$

Using the expression for $r_{\text {end }}$ in (13), we now prove that the $T R T R$ gait results in a decrease of the distance $r$, i.e. $r_{\text {end }}<r_{0}$. The proof holds not only for our specific problem, but assumes a general control system of the form (7) that satisfies the invariance properties (8) and (9). Under these relations, the system is defined solely by the functions $B_{11}^{\prime}(r)$ and $B_{22}^{\prime}(r)$. The proof uses the following assumptions on the system:

A1. $-1<B_{11}^{\prime}(r)<0$ for all $r>r_{c}$.

A2. $\left|B_{22}^{\prime}(r)+1\right|$ decreases monotonously with $r$.

A3. $B_{22}^{\prime}(r)<-1$ for all $r>r_{c}$.

The first two assumptions are fairly general and physically reasonable, as they require that the interactions between the motions of $\mathbf{x}_{p}$ and $\mathbf{x}_{c}$ are monotonously decaying with the distance $r$, and that the magnitude of $\dot{\mathbf{x}}_{p}$ is always less than that of $\dot{\mathbf{x}}_{c}$. The third assumption, which is given here for simplicity, is not essential and can be relaxed. It is easy to verify that the three assumptions hold in our example, where the data of the model is given in (10). The following theorem uses the assumptions to prove that $r$ is decreasing under the $T R T R$ gait.

Theorem 1: Consider a control system of the form (7), which possesses the invariance relations (8) and (9), and satisfies assumptions A1, A2 and A3. Then under initial distance $r(0)=r_{0}>r_{c}$, the TRTR gait results in a final distance $r_{\text {end }}$ that satisfies $r_{\text {end }}<r_{0}$.

Proof: First, we show that $\Delta_{o}$, defined in (13), is positive. Under assumptions $\mathbf{A} 2$ and A3, one obtains

$$
\begin{aligned}
\Delta_{o} & =t_{2}+R_{3}-R_{1}=t_{2}+\frac{r_{2}}{\left|B_{22}^{\prime}\left(r_{2}\right)\right|}-\frac{r_{0}}{\left|B_{22}^{\prime}\left(r_{0}\right)\right|} \\
& >t_{2}+\frac{r_{2}-r_{0}}{\left|B_{22}^{\prime}\left(r_{2}\right)\right|}>t_{2}+r_{2}-r_{0} .
\end{aligned}
$$

Assumption A1 implies that under the differential equation (11), one has $t>r_{0}-\Phi\left(r_{0}, t\right)$. Hence, we conclude that $t_{2}+r_{2}-r_{0}>0$, which implies $\Delta_{o}>0$.

Next, we prove that $t_{4}<t_{2}$. Using the fact that $\Delta_{o}>0$, we have

$$
\begin{aligned}
& \left(\Delta_{o}+R_{1} \cos \theta_{1}\right)^{2}+\left(R_{1} \sin \theta_{1}\right)^{2} \\
& =\left(t_{2}+R_{3}+R_{1}\left(\cos \theta_{1}-1\right)\right)^{2}+\left(R_{1} \sin \theta_{1}\right)^{2} \\
& =\left(t_{2}+R_{3}\right)^{2}+2 R_{1}\left(\cos \theta_{1}-1\right)\left(t_{2}+R_{3}-R_{1}\right) \\
& =\left(t_{2}+R_{3}\right)^{2}+2 R_{1}\left(\cos \theta_{1}-1\right) \Delta_{o}<\left(t_{2}+R_{3}\right)^{2} .
\end{aligned}
$$

Substituting into the expression for $t_{4}$ in (13) yields $t_{4}<t_{2}$.

Finally, assumption A1 implies that $\Phi\left(r_{0}, t\right)$ is monotonously decreasing with $t$. Therefore, using the 

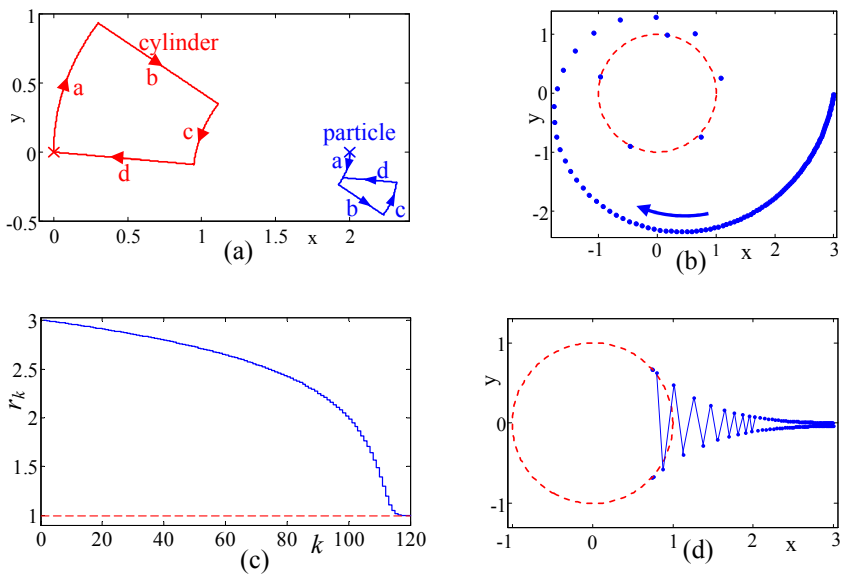

Fig. 4. (a) Trajectories of $\mathbf{x}_{c}$ and $\mathbf{x}_{p}$ under a single gait. (b) Motion of particle $\mathbf{x}_{p}$ under iterative applications of gait. (c) The series $r_{k}$ of the distance $r$ after each cycle. (d) Motion of $\mathbf{x}_{p}$ under iterative applications of the gait with alternating $\theta$-shift.

expression for $r_{\text {end }}$ in (13) and the fact that $t_{4}<t_{2}$ proves that $r_{\text {end }}<r_{0}$.

Example: We now show a simulation example in order to demonstrate the particle motion induced by the TRTR gait. Fig. 4(a) shows the trajectories of $\mathbf{x}_{c}$ and $\mathbf{x}_{p}$ for $r_{c}=1$, under the TRTR gait with $t_{1}=t_{2}=1$. The initial positions, which are marked by ' $x$ ', are $\mathbf{x}_{c}(0)=$ $(0,0)$ and $\mathbf{x}_{p}(0)=(2,0)$. One can clearly see that while $\mathbf{x}_{c}$ travels along a closed loop, $\mathbf{x}_{p}$ has a nonzero shift of $\Delta \mathbf{x}_{p}=(-0.0045,-0.0290)$, or equivalently, $\Delta r=-0.0043$ and $\Delta \theta=-0.55^{\circ}$. When applying the gait repeatedly, the distance $r$ after each cycle is slowly decreasing, and the angle $\theta$ is changing monotonously, resulting in a spiral motion of the particle until it eventually reaches the boundary of the cylinder. Fig. 4(b) shows the position of $\mathbf{x}_{p}$ after each cycle under iterative application of the $T R T R$ gait, starting from $\mathbf{x}_{c}(0)=(0,0)$ and $\mathbf{x}_{p}(0)=(3,0)$. Fig. 4(c) plots the discrete series $r_{k}$ of distances after each iteration. It can be seen that after $k=115$ iterations, the particle is trapped at the cylinder's boundary $r=r_{c}=1$. In order to eliminate the monotonous change of $\theta$ which results in spiral motion, we now modify the sequence of gaits by reversing the direction of the tangential motions along arcs in steps $\mathrm{A}$ and $\mathrm{C}$ alternately between consecutive gaits. This results in oscillations of $\theta_{k}$ around zero, as shown in Fig 4(d). The evolution of the distances $r_{k}$ remains the same as in Fig 4(c).

Remarks: First, note the stopping condition in step C of the algorithm, which assures that the trajectory forms a closed loop. Second, a key fact is that the TRTR gait requires feedback, since it is based on moving $\mathbf{x}_{c}$ in the directions $\mathbf{e}(\mathbf{q})$ or $\mathbf{e}^{\perp}(\mathbf{q})$, which depend on the positions of $\mathbf{x}_{p}$ and $\mathbf{x}_{c}$. However, the feedback does not necessarily require inertial sensors that measure absolute positions, and can be implemented under two much simpler requirements, as follows. The first requirement is the ability to mark the starting point $\mathbf{x}_{c}(0)$ with some fixed landmark. The second requirement is that the moving cylinder is equipped with a compass-like device which is capable of measuring the bearing angle of the line connecting $\mathbf{x}_{c}$ to the particle $\mathbf{x}_{p}$, and of the line connecting it to the the landmark of $\mathbf{x}_{c}(0)$. Finally, it is important to note that the TRTR gait does not require knowledge of the exact details of the model. Therefore, the gait is robust with respect to inaccuracies in the model. Moreover, the gait can be implemented in any system describing the interaction between two circular bodies, as long as their dynamics induces a connection of the form (7) with the invariance properties (8) and (9).

Implementation of the gait in Stokes flow: In order to demonstrate the fact that the TRTR gait is modelindependent, we now show how it can be implemented for a simple example in viscous fluid which is governed by Stokes' equation. Stokes equation describes the hydrodynamics of particles on a small scale, where the Reynolds number is very small, indicating that viscous effects are dominating and inertial effects are negligible [13], [25]. We consider the case of two spheres of equal radius, where the motion of one sphere is controlled while the other sphere moves passively with the fluid. The goal is again to move the controlled sphere in small loops that induce attraction of the passive sphere to the controlled sphere. Note that the motion of rigid bodies in Stokes flow is quasistatic, i.e. the net forces acting on each rigid body are zero at all times. Another fundamental principle is the existence of a linear relation between the rigid-body velocities and the forces exerted by the fluid on the rigid bodies. For two rigid bodies, this linear relation is formulated as

$$
\left(\begin{array}{c}
\mathbf{f}_{1} \\
\mathbf{f}_{2}
\end{array}\right)=\left(\begin{array}{ll}
\mathbf{R}_{11} & \mathbf{R}_{12} \\
\mathbf{R}_{21} & \mathbf{R}_{22}
\end{array}\right)\left(\begin{array}{c}
\dot{\mathbf{x}}_{1} \\
\dot{\mathbf{x}}_{2}
\end{array}\right),
$$

where $\mathbf{f}_{i}$ is the force acting on the $i$ th body, $\dot{\mathbf{x}}_{i}$ is its velocity, and the big matrix with blocks $\mathbf{R}_{i j}$ in (14) is called the resistance matrix [13]. Consider a given motion of one body $\mathbf{u}=\dot{\mathbf{x}}_{1}$, imposed by applying an external force on it. The resulting motion of the passive body $\dot{\mathbf{x}}_{2}$ can be obtained by using the relation (14) along with the quasistatic assumption $\mathbf{f}_{2}=0$, and is thus given by $\dot{\mathbf{x}}_{2}=-\mathbf{R}_{22}^{-1} \mathbf{R}_{21} \dot{\mathbf{x}}_{1}$. This relation is again in the form of a driftless nonlinear control system (7) induced by a connection of the form (6). When the two rigid bodies are spheres, the control system also satisfies the two invariance properties given in (8) and (9), hence we can apply the $T R T R$ gait in a similar fashion. For simplicity, in our simulation we use the far-field approximation of the resistance matrix, which was developed in [9]. We simulated the motion of two spheres of radius 1, where the sphere $\mathbf{x}_{1}$ is controlled and the sphere $\mathbf{x}_{2}$ moves passively. Fig. 5(a) shows trajectories of $\mathbf{x}_{1}(t)$ and $\mathbf{x}_{2}(t)$ under a single $T R T R$ gait with $t_{1}=t_{2}=1$, where the initial positions are $\mathbf{x}_{1}(0)=(0,0)$ and $\mathbf{x}_{2}(0)=(4,0)$. The resulting shift in the position of $\mathbf{x}_{2}$ is $\Delta r=-0.0054$ and $\Delta \theta=-0.96^{\circ}$. One noticeable difference from the cylinder-particle motion in perfect fluid is that the arcs of $\mathbf{x}_{1}$ and $\mathbf{x}_{2}$ in steps $\mathrm{A}$ and $\mathrm{C}$ are in-phase, whereas they were anti-phase in the perfect fluid case of Fig. 4(a). (This is caused by the fact 

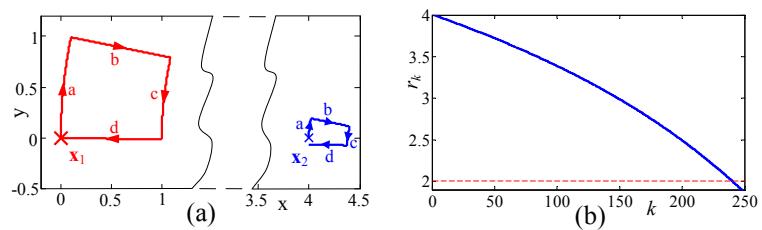

Fig. 5. The motion of two spheres in Stokes flow: (a) Trajectories of $\mathbf{x}_{1}$ and $\mathbf{x}_{2}$ under a single gait. (b) The series $r_{k}$ of the distance $r$ after each cycle.

that here we have $\mathbf{B}_{22}^{\prime}(r)>-1$, while in the perfect fluid case we had $\mathbf{B}_{22}^{\prime}(r)<-1$, see Eq. (10)). When applying the gait repeatedly, the discrete series of distances $r_{k}$ decreases monotonously as shown in Fig. 5(b), and reaches contact $r=2$ after approximately 240 iterations.

Notice the difference in the nature of convergence in the two examples. In the inviscid case, the distance $r_{k}$ converges to zero only asymptotically, and physical contact $r=1$ is never attained. In the second example of Stokes flow, the model predicts convergence to contact $(r=2)$ in finite time. This unphysical result is an artifact, caused by the fact that we have used a far-field approximation of the resistance matrix. This approximation is not valid when the gap between the particles is small, where a more refined model accounting for lubrication effects [19] is needed. An additional fundamental difference between the cases of Stokes flow and potential flow is the fact that in Stokes flow, the no-slip condition on the boundary of the spheres implies that pure rotation of the controlled sphere also leads to motion of the passive sphere, and thus it can serve as an additional input for the control system, which may enhance the manipulation capabilities.

\section{Controllability on Symplectic Fiber BUNDLES}

In this last section, we return to the connection-theoretic treatment of section III. We show that the particle manipulation connection is an example of a symplectic connection, and we establish a controllability test for systems governed by a symplectic connection, which yields the same results as the classical Lie-algebraic test in the Chow theorem [28] but is significantly simpler: whereas the Chow criterion deals with vector fields on the space $B \times F$, our criterion deals with objects on the fiber $F$ only. For the particle manipulation, $\operatorname{dim} B \times F=4$ while $\operatorname{dim} F=2$, which entails a reduction by half. Our result is analogous to that of [18], who developed a criterion called strong controllability for connections on a principal fiber bundle.

The treatment below will make use of differential forms (see [6]). We denote the exterior derivative of a form $\omega$ by $\mathbf{d} \omega$, and the contraction of a vector field $X$ with a form $\omega$ by $\mathbf{i}_{X} \omega$. Recall that a two-form $\omega$ is symplectic when it is closed and non-degenerate, i.e. $\mathbf{d} \omega=0$, and $\mathbf{i}_{X} \omega=0$ implies that $X=0$. A fiber bundle $B \times F$ is said to be symplectic when $F$ is equipped with a symplectic form $\omega_{F}$; see [11].

Definition 3: A connection $\mathbf{A}$ is symplectic if, for each loop in the base space, the associated geometric phase mapping $\Phi$ preserves the symplectic structure $\omega_{F}$ on $F$, i.e.
$\Phi^{*} \omega_{F}=\omega_{F}$.

For the particle manipulation problem, the symplectic form on $F$ is the area form $d x_{p} \wedge d y_{p}$. The connection is symplectic because the fluid is incompressible and hence area-preserving.

Recall that a vector on the trivial bundle $B \times F$ is vertical if it is of the form $\left(0, \dot{\mathbf{x}}_{p}\right)$, where $\dot{\mathbf{x}}_{p} \in T F$, while vectors of the form $\left(\dot{\mathbf{x}}_{c}, \mathbf{A}\left(\mathbf{x}_{p}, \mathbf{x}_{c}\right) \dot{\mathbf{x}}_{c}\right)$ with $\dot{\mathbf{x}}_{c} \in T B$, are said to be horizontal.

Definition 4: A two-form $\omega$ is compatible with a symplectic connection if $\omega(u, v)=0$ for all horizontal vectors $u$ and vertical vectors $v$, and if $\omega(v, w)=\omega_{F}(v, w)$ for all vertical $v, w$, where $\omega_{F}$ is the symplectic form on $F$.

For example, in our problem of particle manipulation, the compatible two-form is given by $\omega=\mathbf{d} \psi_{x} \wedge \mathbf{d} x_{c}+\mathbf{d} \psi_{y} \wedge$ $\mathbf{d} y_{c}+\mathbf{d} x_{p} \wedge \mathbf{d} y_{p}$, where $\psi_{x}, \psi_{y}$ are the elementary stream functions (2).

Using the compatible two-form $\omega$, we define then the following vector spaces of exact one-forms on $F$ :

$$
\begin{array}{ll}
\mathfrak{v}_{1}=\left\{\mathbf{d i}_{X^{h}} \mathbf{i}_{Y h} \omega:\right. & X, Y \in \mathfrak{X}(B)\} \subset T^{*} F \\
\mathfrak{v}_{k}=\left\{\mathbf{d i}_{Z^{h}} \alpha_{k-1}:\right. & \left.Z \in \mathfrak{X}(B), \alpha_{k-1} \in \mathfrak{v}_{k-1}\right\} \subset T^{*} F,
\end{array}
$$

where $k=2,3, \ldots$ Here, $\mathfrak{X}(B)$ denotes the space of vector fields on $B$ and $X^{h}$ is the horizontal lift of a vector field $X$ on $B$, given by $X^{h}\left(\mathbf{x}_{c}, \mathbf{x}_{p}\right)=\left(X\left(\mathbf{x}_{c}\right), \mathbf{A}\left(\mathbf{x}_{p}, \mathbf{x}_{c}\right) \cdot X\left(\mathbf{x}_{c}\right)\right)$. Our controllability result is then as follows.

Theorem 2: Let $\left(\mathbf{x}_{c}, \mathbf{x}_{p}\right)$ be an element of $B \times F$. If the sum of vector spaces

$$
\mathfrak{v}_{1}\left(\mathbf{x}_{c}, \mathbf{x}_{p}\right)+\mathfrak{v}_{2}\left(\mathbf{x}_{c}, \mathbf{x}_{p}\right)+\cdots
$$

coincides with the entire cotangent space $T_{\mathbf{X}_{p}}^{*} F$, then the system is controllable at $\left(\mathbf{x}_{c}, \mathbf{x}_{p}\right)$.

For the particle manipulation problem, we have that $\mathfrak{v}_{1}$ is spanned by $\mathbf{d} H_{1}$ while $\mathfrak{v}_{2}$ is spanned by $\mathbf{d} H_{2,1}$ and $\mathbf{d} H_{2,2}$, where

$$
\begin{gathered}
H_{1}=\frac{r_{c}^{4}}{\left(X^{2}+Y^{2}\right)^{2}}, \quad H_{2,1}=\frac{4 r_{c}^{4} X\left(X^{2}+Y^{2}-r_{c}^{2}\right)}{\left(X^{2}+Y^{2}\right)^{4}}, \\
\text { and } H_{2,2}=\frac{4 r_{c}^{4} Y\left(X^{2}+Y^{2}-r_{c}^{2}\right)}{\left(X^{2}+Y^{2}\right)^{4}} .
\end{gathered}
$$

We recall that $r_{c}$ is the radius of the cylinder. It is easy to check that the span of $\mathbf{d} H_{1}, \mathbf{d} H_{2,1}$ and $\mathbf{d} H_{2,2}$ is $\mathbb{R}^{2}$, except for the points where $X^{2}+Y^{2}=r_{c}^{2}$, corresponding to the boundary of the cylinder. The lack of controllability here reflects the fact that fluid particles on the boundary only move along the boundary and cannot leave it.

Remarks. We defer the proof of the controllability characterization to a future paper. The proof relies on the realization that the functions $H_{1}, H_{2,1}$, etc. are in fact Hamiltonian functions for the iterated Lie brackets that appear in the Chow theorem on controllability. Last, we emphasize that the connection $\mathbf{A}$ for the particle-manipulation problem also enjoys a number of symmetries, which were exploited in the design of gaits. This makes A into a Hannay-Berry connection [21], a type of group-invariant symplectic connection that was 
previously used in the description of averaged equations and Foucault's pendulum.

\section{CONCLUSION}

In this paper, we have studied the motion of a particle in an inviscid, irrotational fluid flow under the influence of a moving rigid body. We have designed a feedback-based gait that results in attraction of the particle, and demonstrated that the gait is model-independent by implementing it for a similar problem in Stokes flow. Moreover, we have shown that this particle drift can be interpreted as the geometric phase of a connection, and by using these geometric structures, we have proposed an alternative controllability criterion with reduced dimensionality.

We now briefly sketch some possible directions for future generalization of the results. We comment first on the extension to the case of three-dimensional fluids. Several remarks are in order here. Firstly, the 3D case is already treated in our example of two spheres in Stokes flow, where a 3D approach is necessary in order to avoid the Stokes paradox (see [13]). We expect that the other examples can easily be extended to the case of spatial fluids too. Mathematically speaking, 3D fluid flows preserve the standard contact structure on $\mathbb{R}^{3}$. As a result, particle manipulation in three dimensions is described by a contact connection, an object whose properties will be described in future work.

Secondly, we plan to investigate optimal gaits that extremize a cost function, such as net motion or overall energy expenditure, under a gait of bounded size. A challenging open problem would be to relate such an optimal gait to a geodesic curve under a suitable metric (see [2], [5]). A third possibility is to consider rigid bodies with non-circular symmetries, such as ellipses, or even fully deformable bodies. Each of these extensions brings in new ways of actuating the fluid. Finally, an long-term goal is to extend these results to the manipulation of many particles in a fluid with a minimal set of control inputs. The geometric description of these extended problems will be the subject of future work.

\section{REFERENCES}

[1] A. Ault, Techniques and Experiments for Organic Chemistry. University Science Books, 1998.

[2] G. A. Araujo and J. Koiller, "Self-propulsion of N-hinged 'animats' at low Reynolds number," Qualitative Theory of Dynamical Systems, vol. 4, pp. 139-167, 2004.

[3] M. D. Armani, S. V. Chaudhary, R. Probst, and B. Shapiro, "Using feedback control of micro-flows to independently steer multiple particles," J. Microelectromechanical Systems, vol. 15, no. 4, pp. 945-956, 2006.

[4] A. Ashkin, Optical Trapping and Manipulation of Neutral Particles Using Lasers. World Scientific, 2007.

[5] J. E. Avron and O. Raz, "A geometric theory of swimming:Purcell's swimmer and its symmetrized cousin," New J. Phys., vol. 10, p. 063016, 2008.

[6] F. Bullo and A. D. Lewis, Geometric control of mechanical systems, ser. Texts in Applied Mathematics. New York: Springer-Verlag, 2005, vol. 49.

[7] W.-L. Chow, "Über Systeme von linearen partiellen Differentialgleichungen erster Ordnung," Math. Ann., vol. 117, pp. 98-105, 1939.

[8] J. Cortes, S. Martinez, J. P. Ostrowski, and K. A. McIsaac, "Optimal Gaits for Dynamic Robotic Locomotion," Int. J. Robotics Res., vol. 20, no. 9, pp. 707-728, 2001.
[9] L. J. Durlofsky, J. F. Brady, and G. Bossis, "Dynamic simulation of hydrodynamically interacting particles," J. Fluid Mech., vol. 180, pp. 21-49, 1987.

[10] S. D. Fleming and R. S. King, Micromanipulation in Assisted Conception: A User's Manual and Troubleshooting Guide. Cambridge University Press, 2003.

[11] V. Guillemin, E. Lerman, and S. Sternberg, Symplectic fibrations and multiplicity diagrams. Cambridge: Cambridge University Press, 1996.

[12] Z. guo Zhou and Z. wen Liu, "Biomimetic Cilia Based on MEMS Technology," J. Bionic Eng., vol. 5, pp. 358-365, 2008.

[13] J. Happel and H. Brenner, Low Reynolds Number Hydrodynamics. New Jersey: Prentice-Hall, 1965.

[14] Z. E. Humes, B. L. Smith, and A. L. Minichiello, "Particle Sorting by Aerodynamic Vectoring," Bulletin of the American Physical Society, vol. 50 , no. $251,2005$.

[15] E. Kanso, J. E. Marsden, C. W. Rowley, and J. B. Melli-Huber, "Locomotion of articulated bodies in a perfect fluid," J. Nonlinear Sci., vol. 15, no. 4, pp. 255-289, 2005.

[16] S. D. Kelly and R. M. Murray, "The Geometry and Control of Dissipative Systems," in Proceedings of the 35th IEEE Control and Decision Conference, 1996, pp. 981-986.

[17] S. D. Kelly and H. Xiong, "Controlled Hydrodynamic Interactions in Schooling Aquatic Locomotion," in Proceedings of the 44th IEEE Conference on Decision and Control, 2005, pp. 3904-3910.

[18] S. D. Kelly and R. Murray, "Geometric Phases and Robotic Locomotion," J. Robotic Systems, vol. 12, no. 6, pp. 417-431, 1995.

[19] S. Kim and S. J. Karrila, Microhydrodynamics. Boston: ButterworthHeinemann, 1991.

[20] H. Lamb, Hydrodynamics. Dover Publications, 1932, 6 Ed.

[21] J. Marsden, R. Montgomery, and T. Ratiu, "Reduction, symmetry, and phases in mechanics," Mem. Amer. Math. Soc., vol. 88, no. 436, pp. iv $+110,1990$.

[22] J. B. Melli, C. W. Rowley, and D. S. Rufat, "Motion planning for an articulated body in a perfect planar fluid," SIAM J. Appl. Dyn. Syst., vol. 5, no. 4, pp. 650-669 (electronic), 2006.

[23] M. Nagai, M. Oishi, N. Sakaki, O. Ducloux, M. Oshima, H. Asai, and H. Fujita, "Application of Vorticella's Feeding Mechanism as a Micromixer," in Proceedings of the IEEE 21st International Conference on Micro Electro Mechanical Systems, 2008, pp. 555-558.

[24] B. Nowakowski, S. T. Smith, B. D. Mulany, and S. C. Woody, "Vortex Machining: Localized Surface Modification Using an Oscillating Fiber Probe," in preparation for Journal of Manufacturing Science.

[25] E. M. Purcell, "Life at low Reynolds number," Am. J. Phys., vol. 45, pp. 3-11, 1977.

[26] G. Schlink, http://www.microbelibrary.org/asmonly/ details.asp?id=1436.

[27] E. A. Shammas, H. Choset, and A. A. Rizzi, "Geometric Motion Planning Analysis for Two Classes of Underactuated Mechanical Systems," Int. J. Robotics Res., vol. 26, no. 10, pp. 1043-1073, 2007.

[28] H. J. Sussmann and V. Jurdjevic, "Controllability of nonlinear systems," J. Differential Equations, vol. 12, pp. 95-116, 1972.

[29] P. Tabeling and S. Cheng, Introduction to Microfluidics. Oxford University Press, 2005.

[30] S. Vogel, Comparative Biomechanics: Life's Physical World. Princeton University Press, 2003.

[31] S. C. Woody, Q. Bian, K. Breuer, S. T. Smith, B. W. Woody, and M. Bauza, http://kellyfish.net/insitutec.mov.

[32] — - "Oscillating Probe System for Inducing Microscale Vortex Flow Field and Measurement of Interaction Dynamics," submitted to Journal of Fluid Mechanics. 